\title{
Klare Verhältnisse auch bei submukös selbstständigen Vestibulumplastiken
}

\author{
Eine Vestibulumplastik ist ein Schleimhaut formender intraoraler Eingriff zur operativen Schaffung und/ \\ oder Verbesserung des Mundvorhofs, auch zur Gingivaverbreiterung. Wie lässt sich das abrechnen?
}

Es handelt sich um die folgenden, ausdrücklich oder logisch überall im Vestibulum möglichen plastischen Eingriffe:

- 3210 GOZ „Beseitigung störende Schleimhautbänder“

(i. D. zirka vier Minuten OP-Zeit)

- 4120 GOZ „koronale/apikale Lappenverlegung“

(i. D. zirka acht Minuten OP-Zeit)

- 3240 GOZ „Vestibulumplastik kleineren Umfangs“

(i. D. zirka 16 Minuten OP-Zeit)

- Ä2677 „selbständige submuköse Vestibulumplastik“

(i. D. zirka 23 Minuten OP-Zeit)

- Ä2675 „partielle Vestibulumplastik“(i. D. zirka 28 Minuten OP-Zeit)

Dreh- und Angelpunkt zur Relationsbewertung ist die 3240 GOZ: Die entscheidende Frage ist in deren Leistungsbeschreibung seit der GOZ-Novellierung 2012 beantwortet, nämlich was unter dem Begriff „kleineren Umfangs“ in einer Kieferhälfte beziehungsweise in einem Frontzahnbereich zu verstehen ist: Das ist ein „Bereich bis zu zwei nebeneinanderliegenden Zähnen“.

Wägt man die „partielle“ Ausdehnung der Ä2675 ab gegen „maximal zwei Zahnbreiten“ der 3240 GOZ und dazu die beiden Vergütungen, dann muss bei einem plastischen Eingriff nach Ä2677 oder Ä2675 ein Bereich von mehr als zwei Zahnbreiten betroffen sein. Auf der Rechnung sind die Ausdehnungsangaben für Ä2675 oder Ä2677 (partielle Schleimhautplastiken) wie etwa „als Region 36-38“ auszuweisen.

Im Rückschluss ist klar, dass die Leistungen nach 3210 und auch 4120 GOZ sich im Bereich von Einzelzähnen abspielen, allerdings beide nur einmal je Kieferhälfte oder Frontzahnbereich, dort aber ortsverschieden auch neben anderen Plastiken.

\section{Was ist eine selbstständige Vestibulumplastik?}

Welche Gebührenziffer wann anzusetzen ist, wäre soweit plausibel geklärt: Aber was ist eine selbstständige Vestibulumplastik und was ist eine submuköse, die bei Ä2677 verlangt ist?

Vereinfacht kann man feststellen, dass der Leistungsumfang der Ä2675 gegenüber der submukösen Plastik nach Ä2677 ca. 20 Prozent umfangreicher sein müsste. Die Bewertung geht davon aus, dass Volllappenbildung inklusive Periostablösung bei der Ä2675 höher zu bewerten ist als nur submuköse Lappenbildung unter Belassung des Periostes bei der Ä2677. Aber wieso „selbstständig“? Bei einer selbstständigen Leistung darf orts-/ausdehnungs- und zeitgleich kein weiterer schleimhautchirurgischer Eingriff stattfinden beziehungsweise berechnet werden. Jeder Osteotomieeingriff zum Beispiel in derselben Lokalisation erfordert und beinhaltet aber Lappenbildung - Volllappenbildung und verunmöglicht somit logisch die Berechnung zumindest einer ausdehnungsidentischen Ä2677 submukösen Plastik.
Ausdehnungsangaben auf der Rechnung sind Pflicht Aber wenn nun die submuköse Plastik übergreifend, also nicht ausdehnungsidentisch mit der „partiellen“ nach Ä2675 und gegebenenfalls nur im Osteotomiebereich volle Schleimhautablösung und -spaltung in Periost und Mukosa stattfindet?

Dann wäre diese submuköse Plastik ausdehnungsverschieden, somit nicht ortsgleich und eigenständig - also selbstständig - berechnungsfähig. Und auch hier wieder: Ausdehnungsangaben auf der Rechnung sind Pflicht und sparen Diskussion.

$\mathrm{Zu}$ beachten ist: Die fachgerechte, operationstechnisch ordnungsgemäße unter anderem spannungsfreie originale Zurückverlagerung eines präparierten Aufklappungslappens ist abschließender Teil der eigentlichen Operationsleistung und nicht gesondert als eigenständige Plastik berechnungsfähig (auch nicht nach Ä2381, Ä2382 „Hautplastiken“).

\section{So könnte die Fallgestaltung ausfallen}

Wurde in Voraussicht eines erhöhten Deckungsbedarfs zum Wundverschluss gleich ein „unüblicher“ (größerer oder atypisch geformter) Lappen gebildet, beispielsweise bei Implantation mit nötiger Schleimhautunterfütterung (9090 GOZ) „wegen Volumenänderung im Alveolaranteil“, dann ist eine Wundverschlussplastik (3100 GOZ) berechnungsfähig.

So könnte die Fallgestaltung aber auch ausfallen: Neben Implantation in regio 36, 37 (2x $9010 \mathrm{GOZ}$ ) xenogene Knochenimplantation/Weichteilunterfütterung (9090 GOZ) mit Materialberechnung (zum Beispiel bovines Knochengranulat), GBRMembran anlegen und fixieren (4138 GOZ), ebenfalls mit Materialienberechnungen (Membran, Pins, ggf. Schaber), dann abschließend Ä2675 (vestibulär gelegene Deckungs- und Gingivaverbreiterungsplastik) im Bereich 35 bis 38 .

Andererseits: Neben einer Augmentation (9100) mit gleichzeitiger Implantation (9010) käme die Berechnung der Weichteilunterfütterung, Membrananlegung und Deckungsplastik wegen Abgeltung nicht in Frage.

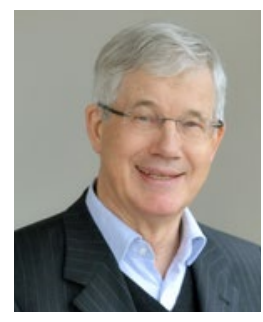

Dr. Peter H. G. Esser

GOZ-Experte und -Berater der ZA eG www.die-za.de 